ARTICLE

https://doi.org/10.1038/s41467-019-13876-x

\title{
Direct observation of dynamic protein interactions involving human microtubules using solid-state NMR spectroscopy
}

\author{
Yanzhang Luo (1) 1,7, ShengQi Xiang (10) 1,2,7, Peter Jan Hooikaas (1) ${ }^{3}$, Laura van Bezouwen ${ }^{4}$, A.S. Jijumon (1) 5,6,

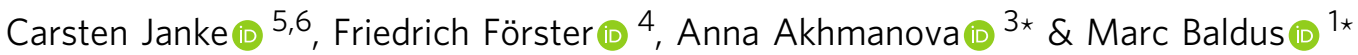

Microtubules are important components of the eukaryotic cytoskeleton. Their structural organization is regulated by nucleotide binding and many microtubule-associated proteins (MAPs). While cryo-EM and X-ray crystallography have provided detailed views of interactions between MAPs with the microtubule lattice, little is known about how MAPs and their intrinsically disordered regions interact with the dynamic microtubule surface. NMR carries the potential to directly probe such interactions but so far has been precluded by the low tubulin yield. We present a protocol to produce $\left[{ }^{13} \mathrm{C},{ }^{15} \mathrm{~N}\right]$-labeled, functional microtubules (MTs) from human cells for solid-state NMR studies. This approach allowed us to demonstrate that MAPs can differently modulate the fast time-scale dynamics of C-terminal tubulin tails, suggesting distinct interaction modes. Our results pave the way for in-depth NMR studies of protein dynamics involved in MT assembly and their interactions with other cellular components.

\footnotetext{
${ }^{1}$ NMR Spectroscopy, Bijvoet Center for Biomolecular Research, Utrecht University, Padualaan 8, 3584 CH Utrecht, The Netherlands. ${ }^{2}$ MOE Key Lab for Membrane-less Organelles \& Cellular Dynamics, School of Life Sciences, University of Science and Technology of China, 96 Jinzhai Road, Hefei 230026 Anhui, China. ${ }^{3}$ Cell Biology, Neurobiology and Biophysics, Department of Biology, Faculty of Science, Utrecht University, Padualaan $8,3584 \mathrm{CH}$ Utrecht, The Netherlands. ${ }^{4}$ Cryo-Electron Microscopy, Bijvoet Center for Biomolecular Research, Utrecht University, Padualaan 8, 3584 CH Utrecht, The Netherlands. ${ }^{5}$ Institut Curie, PSL Research University, CNRS UMR3348, F-91405 Orsay, France. ${ }^{6}$ Université Paris Sud, Université Paris-Saclay, CNRS UMR3348, F-91405 Orsay, France. ${ }^{7}$ These authors contributed equally: Yanzhang Luo, ShengQi Xiang. *email: a.akhmanova@uu.nl; m.baldus@uu.nl
} 
n eukaryotic cells, microtubules (MTs) are cytoskeletal polymers essential for many biological processes, including cell division, migration, polarization, and intracellular trafficking. MTs are assembled from $\alpha / \beta$-tubulin heterodimers and are intrinsically polarized, with the highly dynamic, $\beta$-tubulinexposed plus end, which rapidly switches between growth and shrinkage in a process termed "dynamic instability"1. The dynamic instability of MTs is caused by GTP binding and hydrolysis on tubulin dimers.

Many MT-associated proteins (MAPs) regulate MT structure and function by interacting with MT lattices and/or MT ends ${ }^{2,3}$. Cryo-electron microscopy (EM) has made substantial progress to elucidate the interaction between MAPs and the MT lattice ${ }^{4,5}$, including the cryo-EM reconstruction of the MAP tau binding to $\mathrm{MTs}^{6}$. However, the direct observation of dynamic regions of tubulin, including the unstructured C-terminal tails that are critical for binding of different MT-associated proteins such as tau ${ }^{6}$ has remained challenging due to their intrinsic flexibility. To model such tubulin regions computational approaches were used, for example in the context of tau binding to $\mathrm{MTs}^{6}$ or, more recently, to determine the effect of a-tubulin acetylation on MT structure and stability ${ }^{7}$. Given the wide-spread relevance of protein dynamics for MT function and, in particular, of tubulin tails for cellular processes and human disorders ${ }^{8}$, direct experimental insight into the dynamic interaction of MTs with MAPs is hence of significant interest.

Nuclear magnetic resonance (NMR) spectroscopy has been shown to provide unique structural insights into heterogeneous and dynamical systems at atomic resolution and on different time scales (see, e.g., refs. ${ }^{9-12}$ ). Previously, solid- and solution-state NMR have been used to study the interactions between MTs and isotope-labeled MAPs or small drugs ${ }^{13-19}$. However, these studies were restricted to studying ligands that could be isotope labeled using bacterial expression systems. Thus far, the direct study of functional MTs from human cells that carry NMR-active labeling was precluded due to insufficient protein amounts. In addition, solution-state NMR studies on intact MTs are hampered by protein size and insufficient molecular tumbling. In the following, we describe a solid-state NMR (ssNMR) approach to directly study the interaction of labeled MTs with their binding partners. In particular, this method enables us to probe the dynamics of intact MTs. Moreover, we examine the influence of two different MT-associated proteins upon the C-terminal tail dynamics. We investigate the binding of the CKK domain that is important for the minus-end recognition of the calmodulin-regulated spectrinassociated protein (CAMSAP) ${ }^{15,19}$. Furthermore, we examine the effect of the microtubule-binding domain (MTBD) of the MAP7 family of proteins, which play an important role in regulating kinesin-based intracellular transport and for which the structural details of MT binding are currently unknown ${ }^{20-23}$. Our results suggest that MAP-tubulin tail interactions can involve both fast-time scale interactions between mobile chains as well as slowtime scale binding/unbinding events within stable MT-MAP complexes.

\section{Results}

Functional MTs for ssNMR studies. To obtain milligram quantities of human MTs we used HeLa S3 cells that not only grow as adherent culture but also in suspension, thereby increasing the efficiency of large-scale cultures ${ }^{24}$. In addition, HeLa S3 cells express only a few tubulin isotypes ${ }^{25,26}$ resulting in comparatively homogeneous samples. We isolated tubulin from cell lysates by using a polymerization and depolymerization cycle to remove contaminations (Fig. 1a, b). Subsequently, we polymerized tubulin into MTs with high concentration of 1,4-piperazinediethanesulfonic acid (PIPES) to remove MAPs binding and stabilized MTs with Taxol ${ }^{27}$. As demonstrated by sodium dodecyl sulfate (SDS) polyacrylamide gel electrophoresis, Taxol-stabilized MTs showed a purity of $90 \%$ (Fig. 1b). To check functionality, we incubated our prepared MTs with a fluorescently tagged MTBD of MAP7 domain-containing protein 3 (MAP7D3, in the following abbreviated by MAP7), which served as a MT-binding probe, and found that the purified MTs were decorated by this protein fragment (Supplementary Fig. 1A, B, left). Notably, the binding of MAP7 induced some bundling of MTs (Supplementary Fig. 1A, B). However, the MT bundling does not affect the structure and the dynamics of tubulin $\mathrm{C}$ terminal tails of each individual $\mathrm{MT}^{28}$, and hence did not interfere with our ssNMR experiments presented below. We also investigated whether HeLa S3-derived tubulin can polymerize without Taxol and shows dynamic instability by preparing an additional sample without Taxol, leading to soluble tubulin after a subsequent depolymerization. The resulting tubulin was used in an in vitro reconstitution assay where MTs are polymerized from stable MT seeds with GFP-tagged end binding protein 3 (EB3) as a marker for growing ends. The tubulin we prepared polymerized into MTs and showed phases of growth and shortening (Fig. 1c and Supplementary Fig. 1C, Movie 1), demonstrating that the purified tubulin remains polymerizationcompetent using our protocol. Taken together, this protocol allowed us to purify functional tubulin from mammalian cells that could form MTs, thus providing the basis for our NMR experiments described below.

To obtain isotope-labeled MTs for ssNMR studies, we grew HeLa S3 cells in $2 \mathrm{~L}\left[{ }^{13} \mathrm{C},{ }^{15} \mathrm{~N}\right]$ labeled medium and obtained isotope-labeled, Taxol-MTs (in the following abbreviated by MTs) using the procedure described above. The sample was then packed into a $1.3 \mathrm{~mm}$ MAS NMR rotor and subjected to extended measurement periods using fast MAS rates. To ensure sample integrity under such conditions, we used transmission EM and TIRF microscopy that confirmed that our MT samples remained intact (Fig. 1d and Supplementary Fig. 1B).

MT samples as seen by ${ }^{13} \mathrm{C}$ and ${ }^{31} \mathrm{P}$ ssNMR spectroscopy. To evaluate the sample quality, we first recorded a $2 \mathrm{D}{ }^{13} \mathrm{C}-{ }^{13} \mathrm{C}$ dipolar-based correlation spectrum using radio frequency-driven recoupling $(\mathrm{RFDR})^{29}$ recoupling and compared our data to chemical-shift predictions for MTs (PDB ID: 5SYF) using FANDAS $2.0^{30}$. Overall, the spectrum agreed with in silico estimates (Fig. 2a, left). For example, in $\mathrm{C} \alpha-\mathrm{C} \beta$ regions of serine, threonine and alanine residues, signals were observed in qualitative agreement with predictions for $\alpha$-helical and $\beta$-strand conformations (Fig. 2a, right). Interestingly, alanine signals that were not observed (Fig. 2a, top right) resided in random-coil conformation and related to residues in tubulin loops. The latter observation would be consistent with the occurrence of loop motion as discussed elsewhere ${ }^{7}$ at the experimental temperature $(\sim 298 \mathrm{~K})$ in our dipolar-based experiments. While residue-specific analysis of such effects will require the extension to 3D ssNMR spectroscopy, possibly in combination with amino acid specific labeling ${ }^{11}$, the spectral resolution observed in Fig. 2 for such a large complex hence speaks in favor of a homogenous sample of polymerized MTs.

This notion was further confirmed by ${ }^{31} \mathrm{P}$ NMR signals stemming from nucleotides binding to MTs. In general, $\alpha / \beta$ tubulin dimers contain two nucleotide-binding sites. The GTP molecule bound to a-tubulin is non-hydrolysable, whereas, the nucleotide bound to $\beta$-tubulin is exchangeable, and GTP is hydrolyzed to GDP upon MT polymerization ${ }^{31}$ (Fig. 2b, left). Indeed, we observed ssNMR signals from both GTP and GDP in 
a

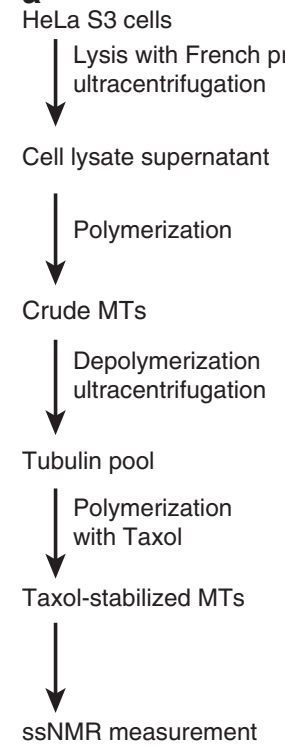

b
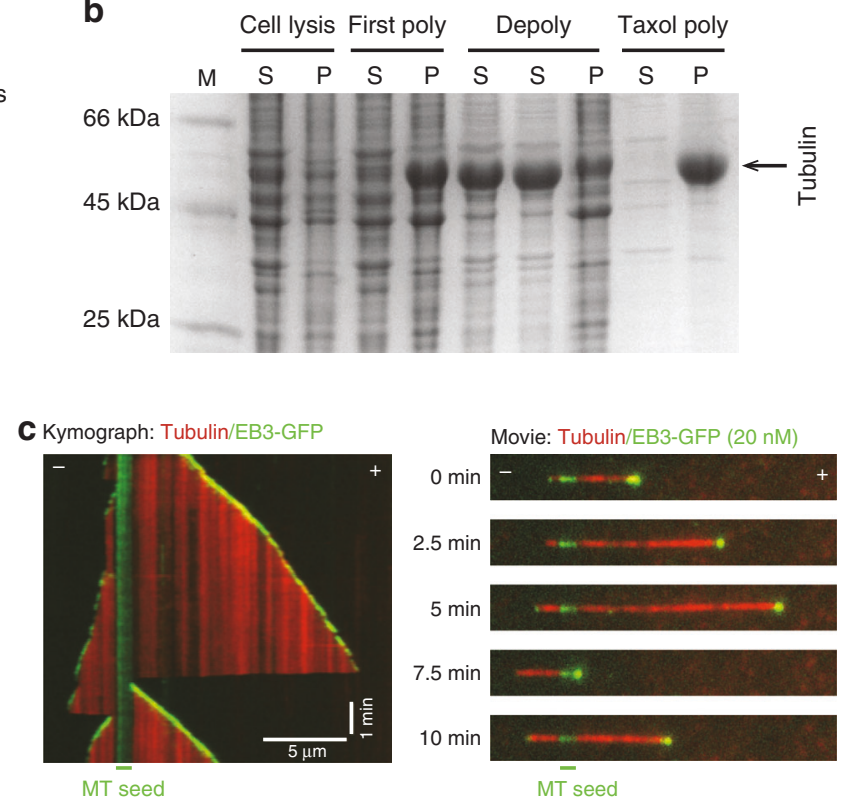
MT seed MT seed

d Before MAS

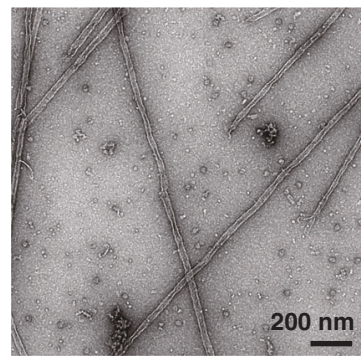

Transmission EM After MAS $44 \mathrm{kHz}$

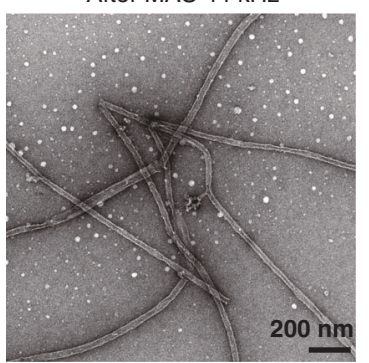

Fig. 1 MT preparation from HeLa S3 cells and sample characterization. a Schematic overview of the sample preparation. b SDS-PAGE analysis from each purification step. M: protein marker; S: supernatant; P: pellet; first poly: first polymerization; depoly: depolymerization; Taxol poly: polymerization with Taxol. The uncropped gel is provided as a Source Data file. c Functional characterization of purified HeLa S3 tubulin. Kymograph (left) and movie stills (right) of an in vitro polymerized MT imaged for $10 \mathrm{~min}$ on a TIRF microscope correspond to Fig. S1C. Time points and MT plus (+) and minus ( - ) ends are indicated. d Characterization of morphology of HeLa MTs before and after MAS NMR experiments by EM.

$1 \mathrm{D}^{31} \mathrm{P}$ CPMAS spectrum (Fig. 2b, right) that were well resolved compared to previous results on lyophilized $\mathrm{MTs}^{32}$. The observed spectral resolution allowed us to distinguish NMR signals from both molecules (Supplementary Table 1) ) $^{32,33}$. Integrated peak intensities (Supplementary Table 1) correlated well with the notion that the molar ratio of GDP:GTP was $1: 1$, which is in agreement with previous studies ${ }^{4,31}$ further supporting the idea that our ssNMR preparations were well folded and functional. Interestingly, we also observed an additional broad peak at $-2.7 \mathrm{ppm}$, which may result from co-purified lipids ${ }^{34}$.

Probing tubulin tails by ssNMR. To probe mobile MT protein segments including the C-terminal tubulin tails (Fig. 3a), we carried out a series of scalar-based (J-based) ssNMR experiments ${ }^{35}$ that previously revealed flexible parts within large biomolecules ${ }^{36-39}$. As discussed elsewhere ${ }^{36,40}$, significant molecular motions with correlation times in the range of $10^{-9}$ to $10^{-7} \mathrm{~s}$ must be present to generate NMR signals in such ssNMR correlation experiments. Slower motions (in the range of milliseconds or slower) will, on the other hand, lead to signal loss (vide infra). In the current context, a $2 \mathrm{D}{ }^{15} \mathrm{~N}-\mathrm{HSQC}$ spectrum contained several signals with a limited spectral dispersion (Fig. 3b), in line with the presence of a mobile unstructured protein region. Using additional $2 \mathrm{D}{ }^{13} \mathrm{C}-\mathrm{HSQC}$ (Fig. $\left.3 \mathrm{c}, \mathrm{d}\right){ }^{15} \mathrm{~N}$ edited ${ }^{1} \mathrm{H}-{ }^{1} \mathrm{H}$ spin diffusion spectra (Fig. $3 \mathrm{~d}$ ), we could confirm that correlations observed in the ${ }^{15} \mathrm{~N}-\mathrm{HSQC}$ spectrum are mainly due to glycine, glutamate, and alanine, which are the most abundant residues in the tubulin tails (Fig. 3a). Moreover, the corresponding resonance frequencies seen in the $2 \mathrm{D}{ }^{13} \mathrm{C}-\mathrm{HSQC}$ reflected random-coil chemical shifts of these amino acids. Note that the latter spectrum contained additional signals from mobile side chains as well as correlations stemming from residual lipids (Fig. 2b) and buffer.

We could further identify correlations stemming from glutamate and alanine residues by tracing $\mathrm{HN}$ to $\mathrm{H} \beta$ or $\mathrm{H} \gamma$ correlations. Interestingly, one glutamate $\mathrm{NH}$ signal showed correlations to the $\mathrm{Ha}$ of glycine, indicating that the corresponding glutamate residues are next to glycine residues (Fig. 3d, second strip). Such correlations are only expected for the $\alpha$ tubulin C-terminal tails (Fig. 3a). On the other hand, the other glutamate peak showed no contacts to glycine $\mathrm{Ha}$ (Fig. 3d, third strip), suggesting that these correlations most likely reflect glutamate residues of the $\beta$-tubulin C-terminal tail (Fig. 3a). In addition, we detected two glycine signals with an approximately 3:1 intensity ratio in line with three glycine residues in EGE motifs of the $\alpha$-tubulin tail and one glycine representing the FGE motif of the $\beta$-tubulin. These tentative assignments were in good 
a
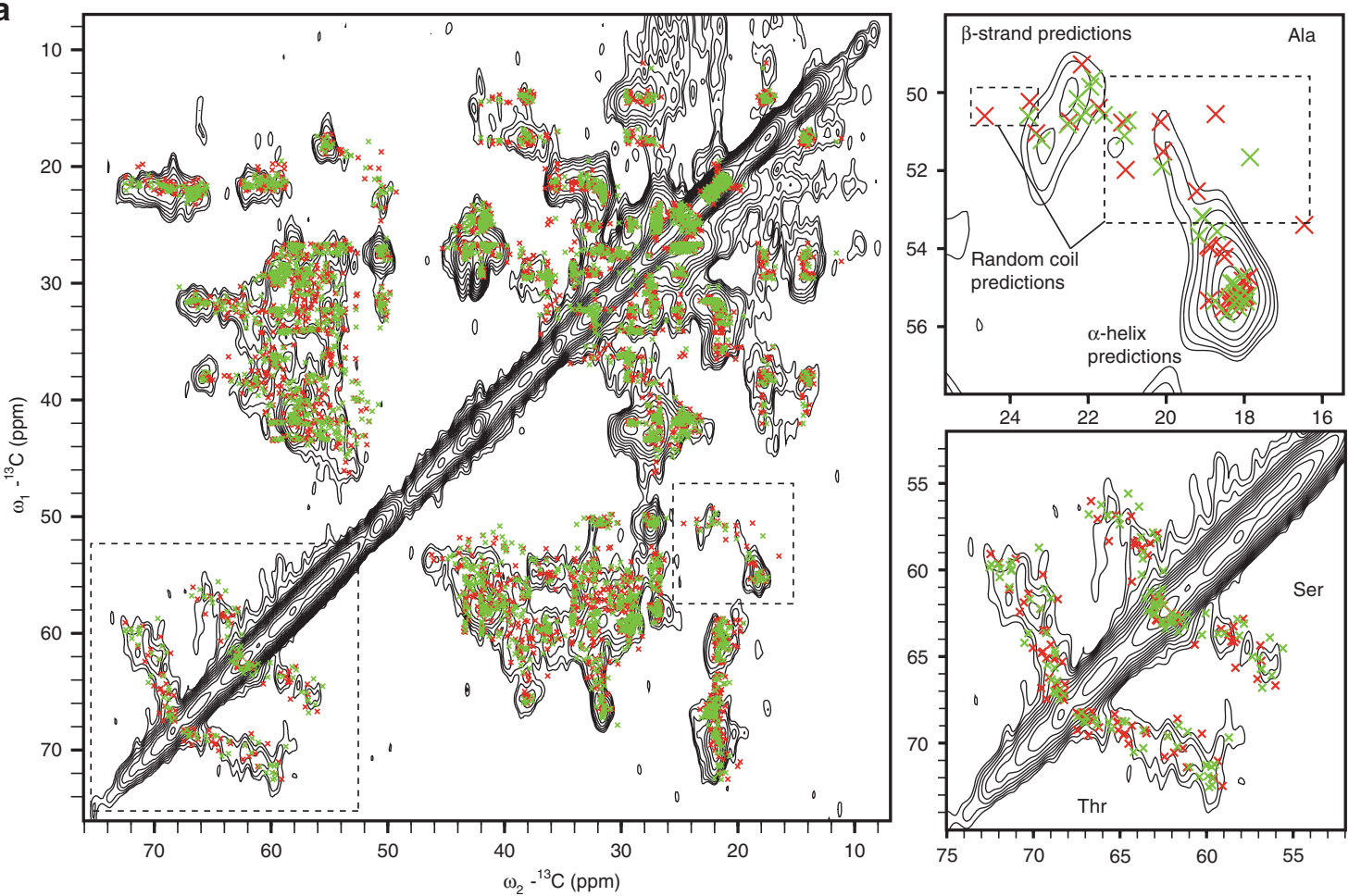

b

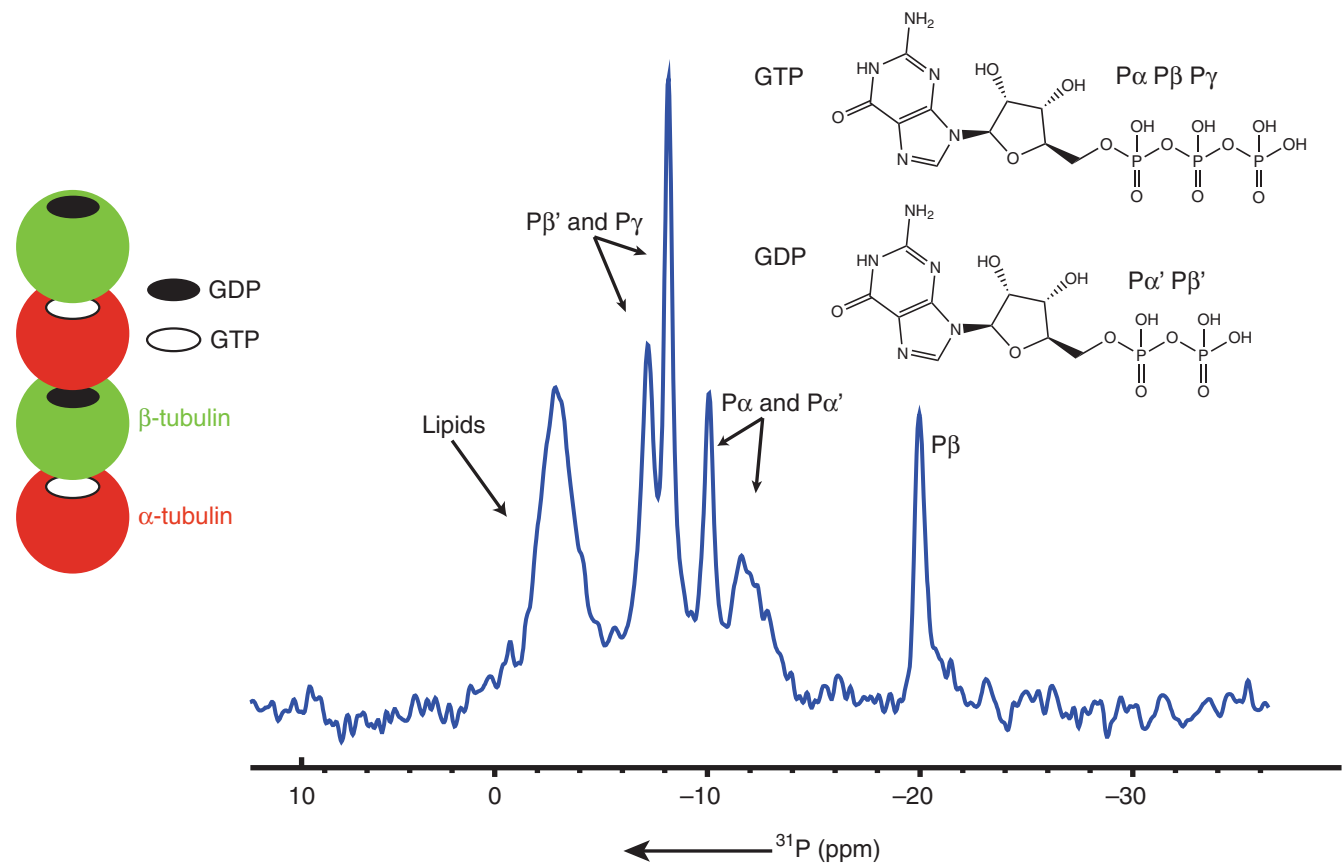

Fig. 2 SsNMR spectra of $\left[{ }^{\mathbf{1 3}} \mathbf{C},{ }^{\mathbf{1 5}} \mathbf{N}\right]$-labeled MTs. a The $2 \mathrm{D}{ }^{13} \mathrm{C}-{ }^{13} \mathrm{C}$ radio frequency-driven recoupling (RFDR) experiment was performed at $270 \mathrm{~K}$ set temperature with a MAS rate of $44 \mathrm{kHz}$ with zoom in's on serine, threonine and alanine $\mathrm{C} \alpha-\mathrm{C} \beta$ regions. Crosses indicate the chemical shift predictions for MTs from SHIFTX2 $2^{57}$ and FANDAS $2.0^{30}$ based on the EM structure (PDB 5SYF). Red and green crosses represent predictions for $\alpha$-tubulin and $\beta$-tubulin, respectively. $\mathbf{b}$ Schematic representation of the GTP/GDP binding to tubulin in MTs (left), and 1D H-31P CP experiment at ambient temperature with MAS rate of $11 \mathrm{kHz}$ allows to observe GTP and GDP bound to MTs.

agreement with previous solution-state NMR studies ${ }^{41}$ of a peptide that contained C-terminal residues of human $\alpha$-tubulin and enabled us to tentatively assign the most C-terminal residue of $\alpha$-tubulin, i.e., Tyr451 (Fig. 3a). Moreover, we could identify one alanine correlation which most likely reflects the last residue of the $\beta$-tubulin based on its high ${ }^{15} \mathrm{~N}$ chemical-shift value, and the correlations from its amide proton to the $\mathrm{H} \beta$ of Ala and Glu.
Lastly, two correlations which may at least in part stem from the $\mathrm{N}$-terminal residues (Fig. 3a) remained unassigned due to lack of cross peaks in the 3D spectrum (Fig. 3b, peaks indicated by asterisks). The corresponding resonance frequencies would speak against posttranslational modifications (PTMs) seen in previous solution-state NMR on Tetrahymena thermophila tubulin ${ }^{42}$. Indeed, earlier work showed that HeLa tubulin mostly lacks 
${ }^{13} \mathrm{C}-\mathrm{HSQC}$

\begin{tabular}{l|l|c} 
& N-terminus & C-terminus \\
\hline$\alpha$-tubulin & MRE & ${ }_{439}$ SVEGEGEEEGEEY $_{451}$ \\
\hline$\beta$-tubulin & MREIV & ${ }_{427}$ DATAEEEEDFGEEAEEEA $_{444}$ \\
\hline
\end{tabular}

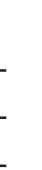

b
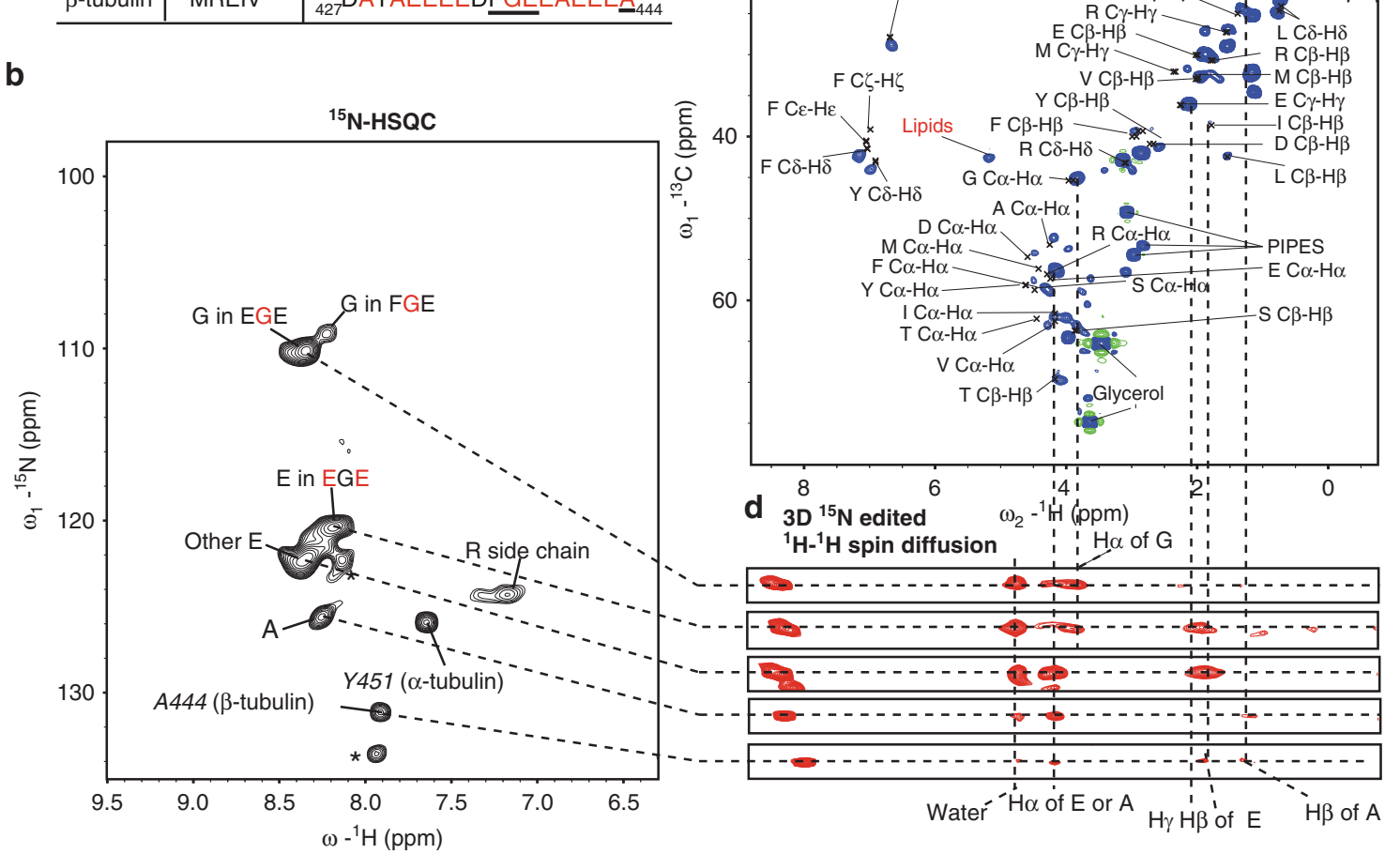

Fig. 3 Flexible tubulin C-terminal investigated by J-based ssNMR. a The sequences of $\mathrm{N}$ - and C-termini of tubulin. Underlined residues showed sequential correlations in our ssNMR data sets. The protein sequences of $\alpha 1$ and $\beta 1$ tubulin isotypes were used for analysis based on previous results 26 . b $2 \mathrm{D}^{15} \mathrm{~N}$-HSQC of $\left[{ }^{13} \mathrm{C},{ }^{15} \mathrm{~N}\right]$-labeled MTs showed the flexible tubulin tails. c $2 \mathrm{D}{ }^{13} \mathrm{C}-\mathrm{HSQC}$ of $\left[{ }^{13} \mathrm{C}\right.$, $\left.{ }^{15} \mathrm{~N}\right]$-labeled MTs showing the residues present in the tubulin tails. Due to the high flexibility, the signals from side chains of leucine, lysine as well as buffer compounds and lipids were also observed. $\mathbf{d}$ Strips of a $3 \mathrm{D}{ }^{15} \mathrm{~N}$-edited ${ }^{1} \mathrm{H}-{ }^{1} \mathrm{H}$ spin diffusion experiment showing the connections between ${ }^{13} \mathrm{C}-\mathrm{HSQC}$ and ${ }^{15} \mathrm{~N}-\mathrm{HSQC}$.

PTMs on tubulin tails ${ }^{43}$. Taken together, our results suggested that both $\alpha$ - and $\beta$-tubulin tails are disordered and highly mobile on a nanosecond time scale.

Tubulin tail dynamics are modulated by the CKK domain. In our previous work we could decipher molecular properties that enable CKK domains of the CAMSAP protein family to preferentially associate with the transition zone between curved protofilaments and the regular MT lattice at MT minus ends ${ }^{15,19}$. We found that the CKK domain that consists of folded protein core and disordered termini associates with the groove between two laterally attached tubulin dimers and discovered that CKKs can subtly differentiate specific tubulin conformations to enable MT minus-end recognition ${ }^{19}$. However, these studies did not reveal how tubulin tails, which are important for CKK binding ${ }^{15}$ are involved in these events. Using our ssNMR protocol, we in the following investigated the influence of CKK binding upon the $\mathrm{C}$ terminal tubulin tails. For this purpose, we purified the unlabeled CAMSAP1 CKK N1492A mutant that exhibits reduced minusend selectivity and binds with higher affinity to MTs compared to wild-type $\mathrm{CKK}^{15,19}$. We then added unlabeled $\mathrm{CKK}$ to $\left[{ }^{13} \mathrm{C}\right.$, ${ }^{15} \mathrm{~N}$ ]-labeled MTs and repeated our ${ }^{15} \mathrm{~N}$-HSQC experiments. Compared to free $\left[{ }^{13} \mathrm{C},{ }^{15} \mathrm{~N}\right]$-labeled MTs, we observed strong changes in the $2 \mathrm{D}$ correlation spectrum (Fig. 4a) that relate to arginine residues of MTs as well as to the C-terminal residues of $\alpha$-tubulin and of $\beta$-tubulin. In the following, we discuss our ssNMR spectral findings and their relationship to previous results for each of these three aspects.
Firstly, signals for the side chains of arginine residues were strongly reduced compared to free MTs. This observation confirmed our previous findings that arginine residues of both $\alpha$ - and $\beta$-tubulin tubulin are located in the binding region of the CKK domain ${ }^{15}$ (Fig. 4b). Furthermore, our recent studies also showed that the binding of CKK induces protofilament skew leading to a reduced lateral space between tubulin dimers ${ }^{15,19}$. Arginine residues located in regions distant from the CKK binding epitope but involved in these lateral interactions between tubulin may hence also become less dynamic ${ }^{44}$.

Secondly, several correlations including the last C-terminal residue of $\beta$-tubulin, A444, disappeared after CKK was bound to MTs and correlations appeared in the alanine, glycine and tyrosine regions (Fig. 4a). These spectral changes would be consistent with conformational exchange on the fast time scale ${ }^{9}$ upon CKK binding. Interestingly, glycines in the FGE segment and alanine residues are only found in the $\mathrm{C}$-terminal tail of $\beta$ tubulin suggesting that the $\beta$-tubulin tail is involved in this dynamic interaction. Notably, the C-termini of both $\beta$-tubulin (17 residues, Fig. 4c) and of the CKK domain (13 residues, Fig. 4c) were not observed in the previous cryo-EM structure ${ }^{15}$. Most likely, the CKK C-terminus hence retains conformational variability upon binding to MTs. Assuming fully extended backbone conformations, both CKK and tubulin C-terminal tails could sample a distance range of at least 60 and $45 \AA^{45}$, respectively, which would readily allow for such dynamic interactions between both mobile tails as indicated in Fig. 4d. Notably, previous EM and ssNMR experiments suggested that the $\mathrm{N}$-terminus of CKK (9 residues) could interact with the C- 
a

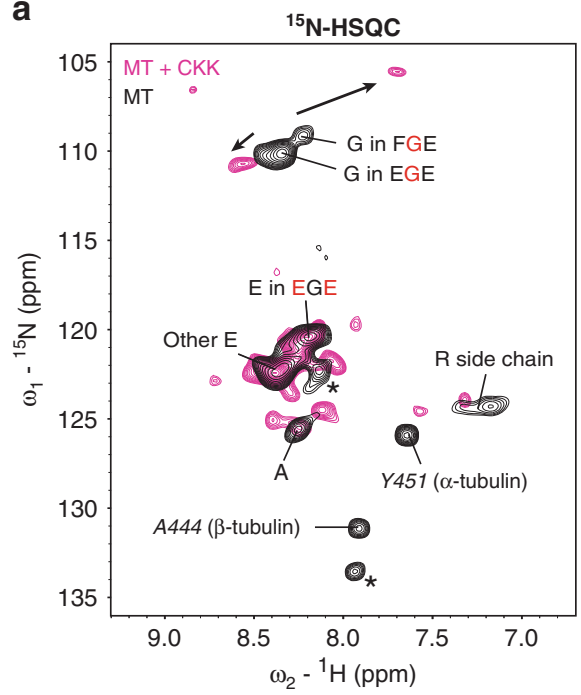

d

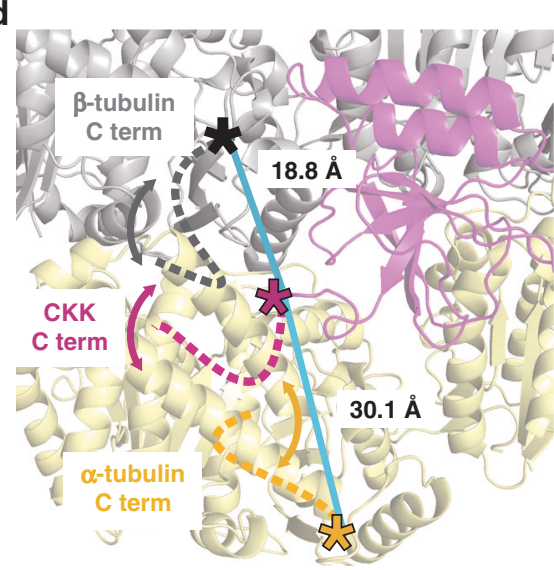

b

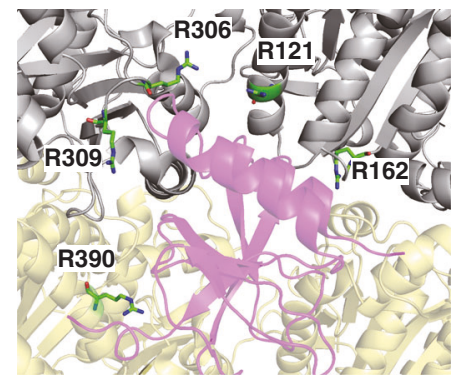

C

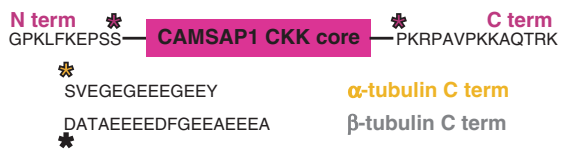

e

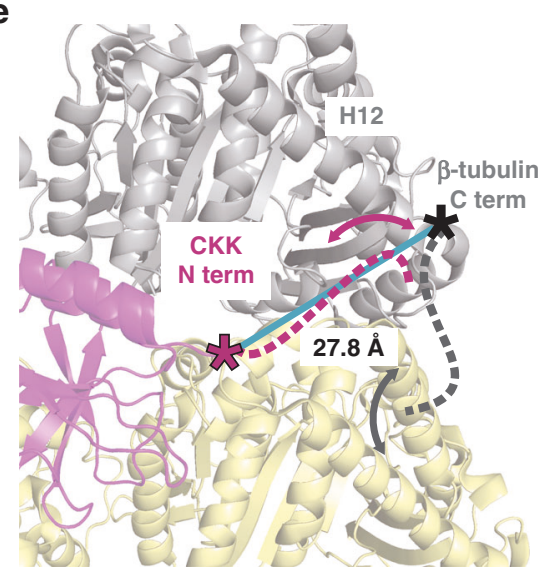

Fig. 4 Tubulin C-terminal tail dynamics are modulated by the CKK domain. a Comparison of $2 \mathrm{D}{ }^{15} \mathrm{~N}-\mathrm{HSQC}$ spectra obtained from $\left[{ }^{13} \mathrm{C}\right.$, $\left.{ }^{15} \mathrm{~N}\right]-$ labeled $\mathrm{MTs}$ (black) and unlabeled CAMSAP1 CKK N1492A in complex with $\left[{ }^{13} \mathrm{C},{ }^{15} \mathrm{~N}\right]$-labeled MTs (magenta). b Zoom-in on arginine residues found in the CKK-MT cryo-EM structure (PDB 5M5C), ref. ${ }^{15}$ ) that are located on $\alpha$-tubulin (yellow) and $\beta$-tubulin (gray) and within $6 \AA$ to CKK domain (magenta). c Top: aminoacid sequences of CKK termini with last residues observed in the cryo-EM structure (PDB 5M5C) indicated by stars. Bottom: C-terminal tails of $\alpha$-tubulin and $\beta$-tubulin with last residues detected the cryo-EM structure (PDB 5M5C) are indicated by yellow and black stars, respectively. $\mathbf{d}$ Dynamic (indicated by double-headed arrows) C-terminal tubulin (dashed black and yellow lines) and CKK (magenta) tails. Blue lines measure the distance between the last residues observed in the cryo-EM structure of both tubulin tails (indicated by black and yellow stars) to the last CKK C-terminal residue observed in cryoEM (magenta star). e Dynamic (indicated by double-headed arrows) CKK N-terminus (dashed magenta line) and $\beta$-tubulin C-terminus (dashed black line). The blue line indicates the distance between the last residue of $\beta$-tubulin and the first residue of CKK detected in the cryo-EM structure.

terminal part of $\beta$-tubulin (Fig. 4c, e), which would be in line with our NMR data.

Thirdly, we also observed changes in ssNMR signal frequencies and intensities after CKK binding for glycine in EGE segments and residue $\mathrm{Y} 451$, respectively, that are both found in the $\mathrm{C}$ terminal tail of $a$-tubulin (Fig. 4a). As shown in Fig. 4c, d, the Cterminal tail of $a$-tubulin with its 12 residues could hence also dynamically interact with the C-terminus of CKK.

Taken together, our results strongly suggest that that the $\mathrm{C}$ terminal tubulin tails dynamically interact with CKK domain on the nano- to microsecond time scale. Combination of these results with our previous EM and ssNMR data further refines this notion, suggesting that the CKK C-terminus interacts with both $\alpha$ - and $\beta$-tubulin while the CKK $\mathrm{N}$-terminus engages in fast interactions with the C-terminus of $\beta$-tubulin. Future ssNMR experiments may allow us to further dissect the details of these dynamic interactions, including the more precise determination of motional time scales in a residue-specific manner.
MAP7-binding reduces MT tail dynamics. The MAP7 family of proteins plays an important role in regulating kinesin-based intracellular transport. However, the structural details of MAP7-MT interaction are currently unknown ${ }^{20-22}$. A secondary-structure prediction of MAP7 on the basis of its amino acid sequence as well as preliminary NMR experiments on free MAP7 in solution suggest that the MAP7 domain adopts a highly a-helical structure separated by a short loop comprising R144 to T146 and flanked by unstructured termini (Fig. 5a).

To obtain insight into the interaction of MT tails with bound MAP7, we repeated our J-based experiments on $\left[{ }^{13} \mathrm{C},{ }^{15} \mathrm{~N}\right]$ labeled MTs after adding unlabeled MAP7 in a 2:1 ratio ensuring full decoration of MTs (see Methods). Interestingly and unlike in the case of binding of the CKK domain to MTs, we did not detect any frequency changes in the $2 \mathrm{D}{ }^{15} \mathrm{~N}-\mathrm{HSQC}$ ssNMR correlation spectrum (Fig. 5b, cyan) compared to the case of free MTs. Instead, signals from both C-terminal tubulin tails, including the identified EGE and FGE segments as well as other E residues were 
a

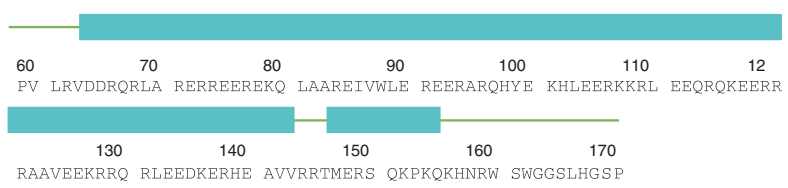

b

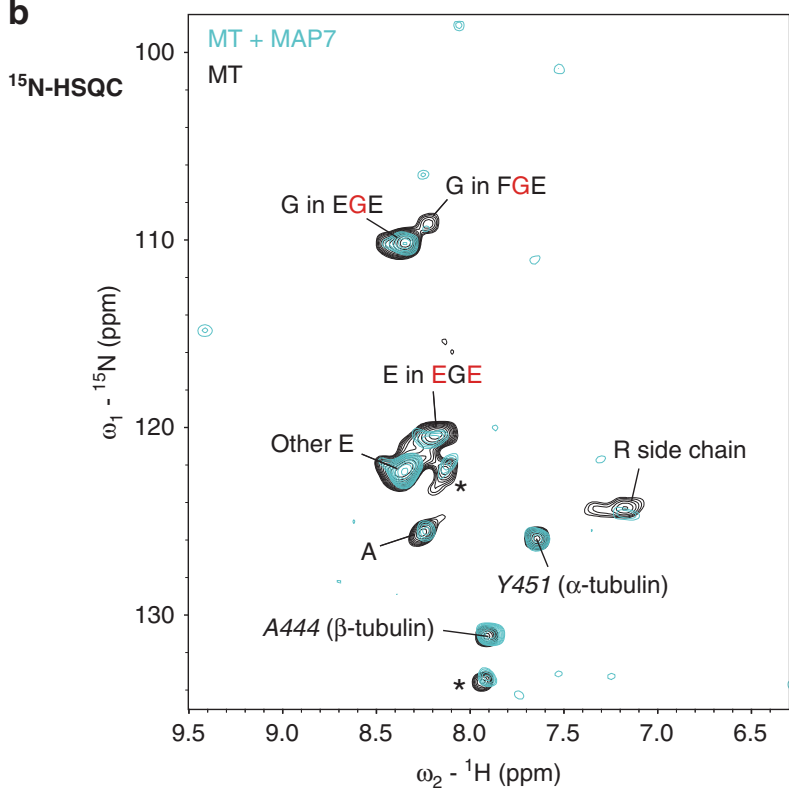

C

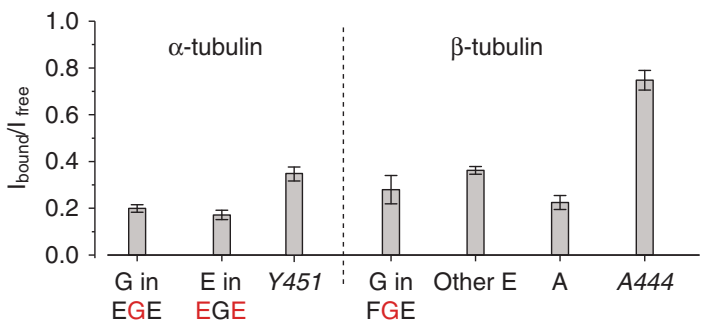

Fig. 5 Tubulin C-terminal tails interact with MAP7. a Secondary structure prediction of MAP7-MTBD by JPred ${ }^{58}$. b Spectral comparison of $2 \mathrm{D}{ }^{15} \mathrm{~N}$ HSQC spectra obtained from $\left[{ }^{13} \mathrm{C},{ }^{15} \mathrm{~N}\right]$-labeled MTs (black) and unlabeled MAP7-MTBD in complex with $\left[{ }^{13} \mathrm{C},{ }^{15} \mathrm{~N}\right]$-labeled MTs (cyan). c Signal intensity ratios of resolved peaks in ${ }^{15} \mathrm{~N}-\mathrm{HSQC}$ between MAP7-MTBD bound MTs and free MTs. Error bars were calculated based on the NMR signal to noise ratio of each peak in the two spectra. Signal intensity ratios are provided as a Source Data file.

strongly attenuated (Fig. $5 \mathrm{c}$ and Supplementary Fig. 2) compared to the most $\mathrm{C}$-terminal residues of $\alpha$ - and $\beta$-tubulin, Y451 and A444, respectively. These observations can be explained by a slow exchange process (millisecond time scale or longer) between freely mobile $\alpha$ - and $\beta$-tubulin tail conformations (visible in the spectra) and C-terminal tails directly bound to MAP7. Note that the latter states would be invisible in our J-based experiments. In a simple two-state model, the relative amount of bound populations would be given by $P_{\text {bound }}=1-I_{\text {bound }} / I_{\text {free }}$ (see, e.g., refs. ${ }^{9,46}$ ) seen in Fig. $5 \mathrm{c}$ leading to values of up to a $80 \%$ bound tail residues around the EGE and FGE terminal segments in $\alpha$ and $\beta$ tubulin tails, respectively, and decreasing to around to a $65 \%$ and $25 \%$ bound population for the most C-terminal residues of $\alpha$ tubulin $\left(\mathrm{Tyr}_{451}\right)$ and of $\beta$-tubulin $\left(\mathrm{Ala}_{444}\right)$, respectively. Unlike the case of $\mathrm{CKK}$ where dynamic tubulin $\mathrm{C}$ termini retain fast dynamics, these observations hence suggested that the MT termini directly bind to MAP7 at a time scale of milliseconds or longer. At the same time the formation of the MT-MAP7 complex also leads to the reduction of Arg side-chain dynamics which are apparent from our experimental data (Fig. 4b) and reminiscent of our findings in the case of CKK binding.

\section{Discussion}

Protein dynamics are critically involved in MT organization including the stochastic switching between growing and shrinking states as well as in the regulation of MTs by MAPs and other cellular factors. NMR carries the potential to directly probe such processes provided that functional and NMR-active MTs can be obtained. We have shown that high-resolution ssNMR spectra can be recorded on $\left[{ }^{13} \mathrm{C},{ }^{15} \mathrm{~N}\right]$ labeled MTs obtained from human cells. The MTs were polymerized from tubulin in functional form $^{24}$, as confirmed by in vitro MT dynamics assays. In addition, our protocol allows obtaining purified, functional tubulin if Taxol is not included in the second polymerization (see Fig. 1c). Such preparations allow for NMR studies of human tubulin in solution (or after depolymerization), e.g., to study MT nucleation and its regulation by different MAPs and potentially related phase transitions ${ }^{47}$. The narrow ${ }^{31} \mathrm{P}$ NMR lines indicated homogenous binding of nucleotides on tubulin subunits that resulted in correctly polymerized MTs. This notion was further confirmed by $2 \mathrm{D}$ dipolar-based correlation experiments that are in qualitative agreement with a MT lattice as seen in cryo-EM structures. The absence of loop signals in our 2D ssNMR dipolar based data possibly points towards enhanced dynamics in protein loop regions as expected for tubulin domains, which are critical for MT dynamic instability or involved in PTMs such as the a-tubulin loop of residues P37-D47 (also known as aK40 loop ${ }^{7}$ ). Further ssNMR studies, possibly using three- or higherdimensional ssNMR experiments may, in combination with tailored labeling, reveal the exact details of these motions.

Complementary to these experiments, proton-detected scalarbased 2D and 3D ssNMR experiments allowed us to directly study the disordered, flexible tubulin tails that are critically involved in MAP binding ${ }^{41}$ and which are invisible in all available high-resolution structures. In the current context, we tracked the effect of binding of the CKK domain of CAMSAP and of MAP7 for the dynamics of $\alpha$ - and $\beta$-tubulin C-terminal tails. Our data suggest that MAP-tubulin tail interactions can take place over a remarkably wide time scale. In the case of CKK, the dynamics of both $\alpha$ - and $\beta$-tubulin C-terminal tails are rapidly (in the nano to microsecond scale) modulated by the CKK domain, confirming our previous finding that tubulin C-terminal tails are important for CKK binding. Combination of these experiments with results of our earlier cryo-EM and ssNMR studies ${ }^{15,19}$ provided further insight regarding the details of these dynamic interactions and suggest that the disordered termini of CKK are involved in these interactions. On the other hand, MAP7 binding to MTs is characterized by tubulin C-terminal tails that exchange between bound (major state) and free (minor state) conformations on a much slower (millisecond or longer) time scale.

The remarkable difference between CKK and MAP7 binding to the tubulin tails may be related in part to the structural and dynamical properties of the MAP itself. For example, the mobile CKK termini comprise several positively charged amino acids (Fig. 4c), whereas, in the case of MAP7, such residues are mostly found in the putatively structured core of MAP7 (Fig. 5a). In addition, the molecular details of the MAP binding epitope on MTs may play a role in defining tail interactions. As we have shown earlier ${ }^{15,19}$, CKK binding takes place between protofilaments. On the other hand, MAP7 is known to compete for binding to $\mathrm{MTs}^{20}$ with the protein tau and hence is likely to be associated with the protofilament crest as seen for tau ${ }^{6}$. Further ssNMR studies may help to dissect these dependencies, including 
the exact CKK and MAP7 regions interacting with the C-terminal tails. Such studies may involve isotope labeled MAPs or mixed labeled $^{48}$ MAP-MT preparations. Moreover, the presented approach could be readily extended to study the interactions of longer variants of MAPs that, in addition to the minimal MT binding region, comprise intrinsically disordered protein domains as well as the Kinesin-1 binding domain ${ }^{22}$. As we have shown recently for the case of mRNA processing bodies ${ }^{39}$, such studies not only could target bona fide MTs but they could also reveal dynamic interactions leading to the compartmentalization of the MT lattice by condensation of tau or other MAPs ${ }^{47,49,50}$. Combination of such NMR experiments with data obtained from cryoEM and fluorescence microscopy experiments could lead to a comprehensive view on the structural and dynamical aspects that define the interaction between MT and MAPs or other cellular players that regulate MT function.

\section{Methods}

Preparation of MTs for ssNMR experiments and in vitro assays. The HeLa S3 cell line (ATCC ${ }^{\oplus}$ CCL2.2 $2^{\text {mix }}$ ) was used for culturing. $\left[{ }^{13} \mathrm{C},{ }^{15} \mathrm{~N}\right]$ labeled DMEM medium was prepared in the same way as described in a previous study ${ }^{51}$ except that we used $3.5 \mathrm{~g} / \mathrm{L}$ glucose in order to maintain cell viability in suspension culture. The cells were first cultured in the labeled medium on two $150 \mathrm{~mm}$ cell culture dishes, and then transferred into 12 dishes with the same medium. When the culture reached a confluence of $\sim 80 \%$ on the plates, cells were trypsinized and transferred into $2 \mathrm{~L}$ labeled medium and cultured in $71 \mathrm{~L}$ Erlenmeyer shaker flasks (Corning) until the cell density reached $\sim 1.2-1.5 \times 10^{6} / \mathrm{mL}$. Cells were then harvested by centrifugation at $500 \times g$ for $20 \mathrm{~min}$ at $4{ }^{\circ} \mathrm{C}$. The cell pellet was collected and resuspended in phosphate-buffered saline and centrifuged again at $500 \times g$ for $15 \mathrm{~min}$ at $4{ }^{\circ} \mathrm{C}$ and used for MT preparation.

The purification of Taxol-stabilized MTs was performed based on the published protocols ${ }^{52,53}$ with minor modifications. Harvested cells were first resuspended with $1 \mathrm{~g}$ cell/mL lysis buffer ( $80 \mathrm{mM}$ PIPES pH 6.8, $1 \mathrm{mM}$ EGTA, $1 \mathrm{mM} \mathrm{MgCl}_{2}$, $1 \mathrm{mM} \beta$-mercaptoethanol, $1 \mathrm{mM}$ PMSF and protease inhibitors (Roche) and lysed on ice by passing through a French Press homogenizer 3 times at 1000 psi. Subsequently, lysed cells were spun down at $120,000 \times g$ at $4{ }^{\circ} \mathrm{C}$ for $30 \mathrm{~min}$ and the supernatant was collected. The supernatant was centrifuged again at $5000 \times g$ at $4{ }^{\circ} \mathrm{C}$ for $15 \mathrm{~min}$ to remove the remaining cell debris and then a half volume of glycerol and $1 \mathrm{mM}$ GTP were added and mixed well. MT polymerization was carried out by incubating the mixture at $30^{\circ} \mathrm{C}$ for $30 \mathrm{~min}$. Subsequently, the crude MT pellet was spun down at $150,000 \times g$ (Type $70.1 \mathrm{Ti}$, Beckman Coulter) at $30^{\circ} \mathrm{C}$ for $30 \mathrm{~min}$ and placed on ice. The pellet was then resuspended in BRB80 buffer ( $80 \mathrm{mM}$ PIPES pH 6.8, $1 \mathrm{mM}$ EGTA, $1 \mathrm{mM} \mathrm{MgCl}_{2}$ ) supplemented with protease inhibitors and kept on ice for $30 \mathrm{~min}$ to allow for MT depolymerization. For a more efficient depolymerization, the solution was resuspended frequently and then centrifuged at $150,000 \times g$ (Type $70.1 \mathrm{Ti}$, Beckman Coulter) at $4{ }^{\circ} \mathrm{C}$ for $30 \mathrm{~min}$, and the supernatant was collected. An equal volume of high concentration PIPES buffer (1 M PIPES pH 6.8, $10 \mathrm{mM} \mathrm{MgCl}_{2}, 20 \mathrm{mM}$ EGTA), together with an equal volume of glycerol and $1 \mathrm{mM} \mathrm{GTP}$ were then added to the supernatant and mixed well. MT polymerization was again performed at $30^{\circ} \mathrm{C}$ for $30 \mathrm{~min}$. Subsequently, $20 \mu \mathrm{M}$ Taxol (Paclitaxel, Sigma) was added to the reaction and incubated for $20 \mathrm{~min}$ to generate Taxol-stabilized MTs. Taxol-stabilized MTs were then spun down at $150,000 \times g\left(\right.$ TLA-55, Beckman Coulter) at $30^{\circ} \mathrm{C}$ for $30 \mathrm{~min}$ washed with BRB80 containing $20 \mu \mathrm{M}$ Taxol and protease inhibitors.

For all in-vitro assays, Taxol-stabilized MTs were prepared in the same way as described above. Regarding the sample used in the in vitro dynamics assay, the preparation of tubulin was similar except that Paclitaxel was not included during the second MT polymerization. After the second MT polymerization, MTs were spun down and resuspended in ice cold BRB80 supplemented with protease inhibitors. The second depolymerization was done on ice for $30 \mathrm{~min}$ and then centrifuged at $150,000 \times g$ at $4^{\circ} \mathrm{C}$ for $30 \mathrm{~min}$. Purified tubulin was aliquoted and snap-frozen in liquid nitrogen, stored at $-80^{\circ} \mathrm{C}$ until use.

In vitro assays. MT seeds were prepared by incubating $20 \mu \mathrm{M}$ porcine tubulin mix containing $70 \%$ unlabeled, $18 \%$ biotin-tubulin and $12 \%$ HiLyte 488 -tubulin with 1 $\mathrm{mM}$ guanylyl- $(\alpha, \beta)$-methylenediphosphonate (GMPCPP) at $37^{\circ} \mathrm{C}$ for $30 \mathrm{~min}$. Polymerized MTs were separated from the mix by centrifugation in an Airfuge for $5 \mathrm{~min}$. MTs were subjected to one round of depolymerization and polymerization in $1 \mathrm{mM}$ GMPCPP, and the final MT seeds were stored in MRB80 buffer $(80 \mathrm{mM}$ K-PIPES pH 6.8, 1 mM EGTA, $4 \mathrm{mM} \mathrm{MgCl}_{2}$ ) containing $10 \%$ glycerol.

In vitro reconstitution assays were performed in flow chambers. Flow chambers were assembled by sticking plasma-cleaned glass coverslips on microscopic slides with double-sided tape. Assays with Taxol-stabilized HeLa MTs were performed in MRB80 prewashed chambers. The in vitro reaction mixture consisted of $15 \mu \mathrm{M}$ HeLa MTs (stabilized by $20 \mu \mathrm{M}$ Taxol) that were either before or after MAS at 44 $\mathrm{kHz}$ for $24 \mathrm{~h}, 150 \mathrm{nM}$ MAP7D3-MTBD, $50 \mathrm{mM} \mathrm{KCl}, 0.1 \%$ Methylcellulose, $0.5 \mathrm{mg} /$ $\mathrm{ml} \kappa$-casein, $1 \mathrm{mM}$ GTP, oxygen scavenging system $(20 \mathrm{mM}$ glucose, $200 \mu \mathrm{g} / \mathrm{ml}$ catalase, $400 \mu \mathrm{g} / \mathrm{ml}$ glucose-oxidase, $4 \mathrm{mM}$ DTT). After centrifugation in an Airfuge for $5 \mathrm{~min}$ at $119,000 \times \mathrm{g}$, the reaction mixture was added to the flow chamber and sealed with vacuum grease.

Dynamic MT assays were performed in flow chambers that were functionalized by sequential incubation with $0.2 \mathrm{mg} / \mathrm{ml}$ PLL-PEG-biotin (Surface Solutions, Switzerland) and $1 \mathrm{mg} / \mathrm{ml}$ NeutrAvidin in MRB80 buffer for $5 \mathrm{~min}$. MT seeds were attached to the biotin-NeutrAvidin links and incubated with $1 \mathrm{mg} / \mathrm{ml} \kappa$-casein. The in vitro reaction mixture consisted of $15 \mu \mathrm{M}$ HeLa tubulin (quantified using bovine serum albumin standard), $20 \mathrm{nM}$ GFP-EB3 and $0.5 \mu \mathrm{M}$ rhodamine-labeled porcine brain tubulin, $50 \mathrm{mM} \mathrm{KCl}, 0.1 \%$ methylcellulose, $0.5 \mathrm{mg} / \mathrm{ml} \kappa$-casein, $1 \mathrm{mM} \mathrm{GTP}$, oxygen scavenging system $(20 \mathrm{mM}$ glucose, $200 \mu \mathrm{g} / \mathrm{ml}$ catalase, $400 \mu \mathrm{g} / \mathrm{ml}$ glucoseoxidase, $4 \mathrm{mM}$ DTT). After centrifugation in an Airfuge for $5 \mathrm{~min}$ at $119,000 \mathrm{~g}$, the reaction mixture was added to the flow chamber containing the HiLyte-488 MT seeds and sealed with vacuum grease. HiLyte488- and rhodamine-tubulin were purchased from Cytoskeleton Inc.

All in vitro experiments were conducted at $30^{\circ} \mathrm{C}$. Data were collected using total internal reflection fluorescence (TIRF) microscopy on an inverted research microscope Nikon Eclipse Ti-E (Nikon) with the perfect focus system (Nikon), equipped with Nikon CFI Apo TIRF $100 \times 1.49$ N.A. oil objective (Nikon, Tokyo, Japan), Photometrics Evolve 512 EMCCD (Roper Scientific) and Photometrics CoolSNAP HQ2 CCD (Roper Scientific) and controlled with MetaMorph 7.7 software (Molecular Devices, CA). The microscope was equipped with TIRF-E motorized TIRF illuminator modified by Roper Scientific France/PICT-IBiSA Institut Curie. For excitation lasers we used $491 \mathrm{~nm} 100 \mathrm{~mW}$ Stradus (Vortran), $561 \mathrm{~nm} 100 \mathrm{~mW}$ Jive (Cobolt) and $642 \mathrm{~nm} 110 \mathrm{~mW}$ Stradus (Vortran). We used an ET-GFP 49002 filter set (Chroma) for imaging of proteins tagged with GFP and an ET-mCherry 49008 filter set (Chroma) for imaging X-rhodamine labeled tubulin amd mCherry-labeled MTBD of MAP7D3. For simultaneous imaging of green and red fluorescence we used an Evolve512 EMCCD camera (Photometrics), ET-GFP/ mCherry filter cube (59022, Chroma) together with an Optosplit III beamsplitter (Cairn Research Ltd.) equipped with double emission filter cube configured with ET525/50m, ET630/75m, and T585lprx (Chroma). To keep in vitro samples at $30^{\circ}$ $\mathrm{C}$, we used a stage top incubator (model INUBG2E-ZILCS; Tokai Hit). Images were processed using ImageJ. All images were modified by linear adjustments of brightness and contrast. Kymographs were generated using ImageJ plugin KymoResliceWide v.0.4. https://github.com/ekatrukha/KymoResliceWide; copy archived at https://github.com/elifesciences-publications/KymoResliceWide).

Transmission EM. Taxol-stabilized MTs were prepared with and without MAS spinning as described above. The protein samples were negatively stained with $2 \%$ uranyl acetate on glow-discharged carbon coated copper grids. Images were recorded on a Tecnai 20 electron microscope with a LaB6 filament, operating at $200 \mathrm{kV}$ with a BM Eagle $4 \mathrm{~K}$ CCD camera (ThermoFisher, Eindhoven, The Netherlands). Images were acquired with a defocus of approximately $5 \mu \mathrm{m}$ The magnification used at $19,000 \times g$ resulting in an effective pixel size of $1.14 \mathrm{~nm}$ on the specimen level.

Preparation of CAMSAP1 CKK N1492A in complex with MTs. $\left[{ }^{13} \mathrm{C},{ }^{15} \mathrm{~N}\right]$ labeled MTs were prepared as described above. The purification of CKK domain and preparation of CKK-MT complexes were performed based on the method published in ref. ${ }^{15}$. CKK was first purified by a ÄKTA pure system with a POROS MC column that was saturated with $\mathrm{Ni}^{2+}$. The column was first equilibrated using washing buffer (50 $\mathrm{mM}$ phosphate buffer, $\mathrm{pH} 8,500 \mathrm{mM} \mathrm{NaCl}, 1 \mathrm{mM} \beta$ mercaptoethanol and $20 \mathrm{mM}$ imidazole). The protein was eluted with the same buffer but containing $400 \mathrm{mM}$ imidazole. Subsequently, the protein was loaded onto a SEC HiLoad Superdex 75 26/60 column (GE Healthcare) equilibrated with $40 \mathrm{mM}$ phosphate buffer, $\mathrm{pH} 7$, supplemented with $150 \mathrm{mM} \mathrm{NaCl}$ and $1 \mathrm{mM}$ DTT. The purified protein was then concentrated and used for ssNMR sample preparation. CKK N1492A was then added to a final concentration of $65.3 \mu \mathrm{M}(4: 1$ $\mathrm{CKK} /$ tubulin) and incubated with labeled MTs at $37^{\circ} \mathrm{C}$ for $30 \mathrm{~min}$. The pellet was centrifuged down at $180,000 \times g$ (Beckman TLA -55 rotor) at $30^{\circ} \mathrm{C}$ for $30 \mathrm{~min}$ and washed with $\mathrm{BRB} 80$, without disturbing the pellet. The pellet was then transferred and packed into a $1.3 \mathrm{~mm}$ rotor.

Preparation of MAP7-MTBD and of MAP7-MTBD- MT complexes. The cDNA of human MAP7-MTBD (residues 59-170) was cloned into the pLICHIS vectors with the gene encoding a $\mathrm{N}$-terminal His tag-Maltose-binding protein (MBP)thrombin cleavage site by using ligation independent cloning ${ }^{54}$.Transformation was done with Escherichia coli Rosetta 2 cells and grown in 1 L unlabeled M9 medium. Induction of the protein was done when $\mathrm{OD}_{600}$ reached 0.6 with $0.3 \mathrm{mM}$ IPTG at $20^{\circ} \mathrm{C}$ for $16 \mathrm{~h}$. The cultures were then centrifuged with $4000 \times g$ at $4{ }^{\circ} \mathrm{C}$ for $20 \mathrm{~min}$ to harvest cells. Cell pellets were washed with $50 \mathrm{mM}$ sodium phosphate buffer, pH $8,500 \mathrm{mM} \mathrm{NaCl}, 1 \mathrm{mM} \beta$-mercaptoethanol and $20 \mathrm{mM}$ imidazole and store at $-80^{\circ} \mathrm{C}$.

For protein purification, cell lysis was done by sonication on ice and the cell lysate was collected by centrifugation of $40,000 \times g$ at $4{ }^{\circ} \mathrm{C}$ for $30 \mathrm{~min}$. Subsequently, the proteins were purified by a ÄKTA pure system with a POROS MC column that was saturated with $\mathrm{Ni}^{2+}$. The column was first equilibrated with 
the same buffer as above mentioned. The cell lysate was then loaded onto the column and the column was washed with 20 column volumes with the same buffer. Proteins were eluted with $50 \mathrm{mM}$ sodium phosphate buffer, $\mathrm{pH} 8,150 \mathrm{mM} \mathrm{NaCl}, 1$ $\mathrm{mM} \beta$-mercaptoethanol and $400 \mathrm{mM}$ imidazole supplemented with protease inhibitors. The protein was concentrated and then diluted with $40 \mathrm{mM}$ sodium phosphate buffer, $\mathrm{pH} 6.5$ to reach a final concentration of imidazole of 20-30 mM. Subsequently, a cation exchange purification was performed with the Hitrap HP SP chromatography column (GE Healthcare Life Sciences). The column was first equilibrated with buffer A ( $40 \mathrm{mM}$ sodium phosphate buffer, $\mathrm{pH} 6.5$ ). The sample was then loaded onto the column and washed 5 column volumes with buffer A. A gradient elution was used by combining buffer A and buffer B ( $40 \mathrm{mM}$ sodium phosphate buffer, $1 \mathrm{M} \mathrm{NaCl}, \mathrm{pH}$ 6.5) to elute the protein. The protein was then concentrated and stored at $4{ }^{\circ} \mathrm{C}$. Finally, thrombin was used to cleave the MBP fusion and then supplemented with protease inhibitors.

A $10 \mathrm{mg} / \mathrm{ml}$ porcine brain tubulin was diluted in BRB80 buffer $(80 \mathrm{mM} \mathrm{K}$ PIPES, pH 6.8, $1 \mathrm{mM}$ EGTA, $1 \mathrm{mM} \mathrm{MgCl}_{2}$ ) to $2 \mathrm{mg} / \mathrm{ml}$. After the addition of $1 \mathrm{mM}$ GTP, the sample was incubated on ice for $5 \mathrm{~min}$. MT polymerization was performed at $30^{\circ} \mathrm{C}$ for $20 \mathrm{~min}$. Subsequently, $20 \mu \mathrm{M}$ taxol was added and incubated for $15 \mathrm{~min}$ at $30^{\circ} \mathrm{C}$. MT pelleting assays in the presence of MAP7-MTBD were performed by mixing with different molar ratios of MAP7-MTBD:MTs. As control treatments, taxol-stabilized MTs or MAP7-MTBD was applied alone. All the samples were then centrifuged at $180,000 \times g$ for $30 \mathrm{~min}$ at $30^{\circ} \mathrm{C}$, an aliquot was taken from the supernatant. After removal of the supernatant, the pellet was resuspended in SDS sample buffer. Samples of supernatant and pellet fractions were loaded and analyzed on Coomassie-stained $12.5 \%$ SDS gels.

Before sample preparation for ssNMR, the purified unlabeled MBP-MAP7MTBD was first incubated with $5 \mathrm{U}$ thrombin at $4{ }^{\circ} \mathrm{C}$ for $16 \mathrm{~h}$ to cleave the fused MBP protein. Protease inhibitors were then added to the proteins. To prepare MAP7-MTBD-MT complexes, $2 \mathrm{mg}$ of purified $\left[{ }^{13} \mathrm{C},{ }^{15} \mathrm{~N}\right]$ labeled MTs were prepared and resuspended in warm BRB80 buffer with $20 \mu \mathrm{M}$ taxol. MAP7-MTBD was then added to molar ratio of 2:1 for MAP7-MTBD:MT and incubated at $30^{\circ} \mathrm{C}$ for $30 \mathrm{~min}$. The pellet was centrifuged down at $180,000 \times g$ (Beckman TLA-55 rotor) at $30^{\circ} \mathrm{C}$ for $30 \mathrm{~min}$ and washed with warm BRB80 buffer with $20 \mu \mathrm{M}$ taxol and protease inhibitors, without disturbing the pellet. The pellet was then transferred and packed into a $1.3 \mathrm{~mm}$ rotor.

ssNMR experiments. NMR experiments of $\left[{ }^{13} \mathrm{C},{ }^{15} \mathrm{~N}\right]$ labeled MTs (free MTs, CKK-bound MTs and MAP7-bound MTs) were performed on a standard-bore $700 \mathrm{MHz}$, a wide-bore $500 \mathrm{MHz}$ (Bruker Biospin). SsNMR experiments on 700 $\mathrm{MHz}$ included 2D ${ }^{13} \mathrm{C}-{ }^{13} \mathrm{C}$ RFDR ${ }^{29},{ }^{1} \mathrm{H}-{ }^{13} \mathrm{C} /{ }^{15} \mathrm{~N}$ HSQC and $3 \mathrm{D}{ }^{15} \mathrm{~N}$-edited ${ }^{1} \mathrm{H}-$ ${ }^{1} \mathrm{H}$ spin diffusion experiments ${ }^{35}$ (set temperature $270 \mathrm{~K}$, MAS rate $44 \mathrm{kHz}$ ). The actual temperature during the ssNMR experiments for both apo as well as MAPbound microtubular samples was $293 \mathrm{~K}$. The ${ }^{13} \mathrm{C}-{ }^{13} \mathrm{C}$ mixing was $3 \mathrm{~ms}$ in the RFDR experiment. The PISSARRO decoupling ${ }^{55}$ scheme of $120 \mathrm{kHz}$ was employed on the ${ }^{1} \mathrm{H}$ channel during the RFDR mixing and the detection of ${ }^{13} \mathrm{C}$. The proton spin diffusion time was $200 \mathrm{~ms}$. In all proton-detected experiments, the PISSARRO decoupling scheme was applied at $11 \mathrm{kHz}$ on ${ }^{1} \mathrm{H},{ }^{13} \mathrm{C}$ and ${ }^{15} \mathrm{~N}$ channels. ${ }^{31} \mathrm{P}$ NMR experiment was conducted on the $500 \mathrm{MHz}$ spectrometer (set temperature $290 \mathrm{~K}$, MAS rate $11 \mathrm{kHz}$ ). The CP MAS experiment was recorded using a $100-50 \%$ ramp on ${ }^{1} \mathrm{H}$-channel of $95.4 \mathrm{kHz}$, and $71.7 \mathrm{kHz}$ on the ${ }^{31} \mathrm{P}$-channel, with $1.2 \mathrm{~ms} \mathrm{CP}$ contact time. A SPINAL decoupling ${ }^{56}$ of ${ }^{1} \mathrm{H}$ during the acquisition of ${ }^{31} \mathrm{P}$ was applied at $90 \mathrm{kHz} .{ }^{31} \mathrm{P}$ chemical shifts were referenced externally using phosphate buffer, $\mathrm{pH} 7$ and set the signal at $0 \mathrm{ppm}$.

Reporting summary. Further information on research design is available in the Nature Research Reporting Summary linked to this article.

\section{Data availability}

Data supporting the findings of this manuscript are available from the corresponding authors upon reasonable request. A reporting summary for this Article is available as a Supplementary Information file. The source data underlying Figs. $1 \mathrm{~b}$ and $5 \mathrm{c}$ and Supplementary Figs. 2 and 3 are provided in a Source Data file.

Received: 30 July 2019; Accepted: 4 December 2019;

Published online: 02 January 2020

\section{References}

1. Desai, A. \& Mitchison, T. J. Microtubule polymerization dynamics. Annu. Rev. Cell Dev. Biol. 13, 83-117 (1997).

2. Akhmanova, A. \& Steinmetz, M. O. Tracking the ends: a dynamic protein network controls the fate of microtubule tips. Nat. Rev. Mol. Cell Biol. 9 , 309-322 (2008).

3. Akhmanova, A. \& Hoogenraad Casper, C. Microtubule minus-end-targeting proteins. Curr. Biol. 25, R162-R171 (2015)
4. Alushin, G. M. et al. High-Resolution microtubule structures reveal the structural transitions in $\alpha \beta$-tubulin upon GTP hydrolysis. Cell 157, 1117-1129 (2014).

5. Nogales, E. \& Zhang, R. Visualizing microtubule structural transitions and interactions with associated proteins. Curr. Opin. Struct. Biol. 37, 90-96 (2016).

6. Kellogg, E. H. et al. Near-atomic model of microtubule-tau interactions. Science 360, 1242-1246 (2018).

7. Eshun-Wilson, L. et al. Effects of a-tubulin acetylation on microtubule structure and stability. Proc. Natl Acad. Sci. 116, 10366-10371 (2019).

8. Gadadhar, S., Bodakuntla, S., Natarajan, K. \& Janke, C. The tubulin code at a glance. J. Cell Sci. 130, 1347-1353 (2017).

9. Peng, J. W. Exposing the moving parts of proteins with NMR spectroscopy. J. Phys. Chem. Lett. 3, 1039-1051 (2012).

10. Wright, P. E. \& Dyson, H. J. Intrinsically disordered proteins in cellular signalling and regulation. Nat. Rev. Mol. Cell Biol. 16, 18 (2014).

11. Renault, M., Cukkemane, A. \& Baldus, M. Solid-state NMR spectroscopy on complex biomolecules. Angew. Chem. Int. Ed. 49, 8346-8357 (2010).

12. Quinn, C. M. \& Polenova, T. Structural biology of supramolecular assemblies by magic-angle spinning NMR spectroscopy. Q. Rev. Biophys. 50, e1 (2017).

13. Kumar, A. et al. Interaction of epothilone B (Patupilone) with microtubules as detected by two-dimensional solid-state NMR spectroscopy. Angew. Chem. Int. Ed. 49, 7504-7507 (2010).

14. Yan, S. et al. Atomic-resolution structure of the CAP-Gly domain of dynactin on polymeric microtubules determined by magic angle spinning NMR spectroscopy. Proc. Natl Acad. Sci. 112, 14611-14616 (2015).

15. Atherton, J. et al. A structural model for microtubule minus-end recognition and protection by CAMSAP proteins. Nat. Struct. Mol. Biol. 24, 931-943 (2017).

16. Kubo, S. et al. A gel-encapsulated bioreactor system for NMR studies of protein-protein interactions in living mammalian cells. Angew. Chem. Int. Ed. 52, 1208-1211 (2013).

17. Kesten, C. et al. The companion of cellulose synthase 1 confers salt tolerance through a Tau-like mechanism in plants. Nat. Commun. 10, 857 (2019).

18. Kadavath, H. et al. The binding mode of a tau peptide with tubulin. Angew. Chem. Int. Ed. 57, 3246-3250 (2018).

19. Atherton, J. et al. Structural determinants of microtubule minus end preference in CAMSAP CKK domains. Nat. Commun. 10, 5236 (2019).

20. Monroy, B. Y. et al. Competition between microtubule-associated proteins directs motor transport. Nat. Commun. 9, 1487 (2018).

21. Chaudhary, A. R. et al. MAP7 regulates organelle transport by recruiting kinesin-1 to microtubules. J. Biol. Chem. 294, 10160-10171 (2019).

22. Pan, X. et al. MAP7D2 localizes to the proximal axon and locally promotes kinesin-1-mediated cargo transport into the axon. Cell Rep. 26, 1988-1999. e1986 (2019).

23. Hooikaas, P. J. et al. MAP7 family proteins regulate kinesin-1 recruitment and activation. J. Cell Biol. 218, 1298-1318 (2019).

24. Souphron, J. et al. Purification of tubulin with controlled post-translational modifications by polymerization-depolymerization cycles. Nat. Protoc. 14, 1634-1660 (2019).

25. Newton, C. N. et al. Intrinsically slow dynamic instability of HeLa cell microtubules in vitro. J. Biol. Chem. 277, 42456-42462 (2002).

26. Davis, A., Martinez, S., Nelson, D. \& Middleton, K. A tubulin polymerization microassay used to compare ligand efficacy. Methods Cell Biol. 95, 331-351 (2010).

27. Weaver, B. A. \& Bement, W. How taxol/paclitaxel kills cancer cells. Mol. Biol. Cell 25, 2677-2681 (2014)

28. Walczak, C. E. \& Shaw, S. L. A. MAP for bundling microtubules. Cell 142, 364-367 (2010).

29. Bennett, A. E. et al. Homonuclear radio frequency-driven recoupling in rotating solids. J. Chem. Phys. 108, 9463-9479 (1998).

30. Narasimhan, $\mathrm{S}$ et al. Rapid prediction of multi-dimensional NMR data sets using FANDAS. In Protein NMR: Methods and Protocols (ed. Ghose, R.) (Springer, New York, 2018).

31. Menéndez, M., Rivas, G., Díaz, J. F. \& Andreu, J. M. Control of the structural stability of the tubulin dimer by one high affinity bound magnesium ion at nucleotide N-site. J. Biol. Chem. 273, 167-176 (1998).

32. Hyang, L. G. et al. Solid-state 31P NMR investigation on the status of guanine nucleotides in paclitaxel-stabilized microtubules. Magn. Reson. Chem. 53, 330-336 (2015)

33. Spoerner, M. et al. High pressure 31P NMR spectroscopy on guanine nucleotides. J. Biomol. NMR 67, 1-13 (2017).

34. Kaur, H. et al. Coupled ATPase-adenylate kinase activity in ABC transporters Nat. Commun. 7, 13864 (2016).

35. Alam, T. M. \& Holland, G. P. 1H-13C INEPT MAS NMR correlation experiments with $1 \mathrm{H}-1 \mathrm{H}$ mediated magnetization exchange to probe organization in lipid biomembranes. J. Magn. Reson. 180, 210-221 (2006). 
36. Andronesi, O. C. et al. Determination of membrane protein structure and dynamics by magic-angle-spinning solid-state NMR spectroscopy. J. Am. Chem. Soc. 127, 12965-12974 (2005).

37. Ward, M. E. et al. Proton detection for signal enhancement in solid-state NMR experiments on mobile species in membrane proteins. J. Biomol. NMR 63, 375-388 (2015).

38. Xiang, S. et al. Site-specific studies of nucleosome interactions by solid-state NMR spectroscopy. Angew. Chem. Int. Ed. 57, 4571-4575 (2018).

39. Damman, R. et al. Atomic-level insight into mRNA processing bodies by combining solid and solution-state NMR spectroscopy. Nat. Commun. 10, 4536 (2019).

40. Schmidt-Rohr K., Spiess H. W. Multidimensional Solid-State NMR and Polymers. (Academic Press, 1994).

41. Lefèvre, J. et al. The $\mathrm{C}$ terminus of tubulin, a versatile partner for cationic molecules: Binding of tau, polyamines, and calcium. J. Biol. Chem. 286, 3065-3078 (2011).

42. Wall, K. P. et al. Molecular determinants of tubulin's C-terminal tail conformational ensemble. ACS Chem. Biol. 11, 2981-2990 (2016).

43. Barisic, M. et al. Microtubule detyrosination guides chromosomes during mitosis. Science 348, 799 (2015).

44. Zhang, R., Alushin Gregory, M., Brown, A. \& Nogales, E. Mechanistic origin of microtubule dynamic instability and its modulation by EB proteins. Cell 162, 849-859 (2015).

45. Ainavarapu, S. R. K. et al. Contour length and refolding rate of a small protein controlled by engineered disulfide bonds. Biophys. J. 92, 225-233 (2007).

46. Lakomek, N.-A., Yavuz, H., Jahn, R. \& Pérez-Lara, Á. Structural dynamics and transient lipid binding of synaptobrevin-2 tune SNARE assembly and membrane fusion. Proc. Natl Acad. Sci. 116, 8699-8708 (2019).

47. Hernández-Vega, A. et al. Local nucleation of microtubule bundles through tubulin concentration into a condensed tau phase. Cell Rep. 20, 2304-2312 (2017).

48. Etzkorn, M., Bockmann, A., Lange, A. \& Baldus, M. Probing molecular interfaces using $2 \mathrm{D}$ magic-angle-spinning NMR on protein mixtures with different uniform labeling. J. Am. Chem. Soc. 126, 14746-14751 (2004).

49. Siahaan, V. et al. Kinetically distinct phases of tau on microtubules regulate kinesin motors and severing enzymes. Nat. Cell Biol. 21, 1086-1092 (2019).

50. Tan, R. et al. Microtubules gate tau condensation to spatially regulate microtubule functions. Nat. Cell Biol. 21, 1078-1085 (2019).

51. Kaplan, M. et al. EGFR dynamics change during activation in native membranes as revealed by NMR. Cell 167, 1241-1251.e1211 (2016).

52. Nirschl Jeffrey, J., Magiera Maria, M., Lazarus Jacob, E., Janke, C. \& Holzbaur Erika, L. F. $\alpha$-Tubulin tyrosination and CLIP-170 phosphorylation regulate the initiation of dynein-driven transport in neurons. Cell Rep. 14, 2637-2652 (2016).

53. Guedes-Dias, P. et al. Kinesin-3 responds to local microtubule dynamics to target synaptic cargo delivery to the presynapse. Curr. Biol. 29, 262-282.e8 (2019).

54. de Jong, R. N., Daniëls, M. A., Kaptein, R. \& Folkers, G. E. Enzyme free cloning for high throughput gene cloning and expression. J. Struct. Funct. Genomics 7, 109-118 (2006).

55. Weingarth, M., Trebosc, J., Amoureux, J. P., Bodenhausen, G. \& Tekely, P. Efficiency at high spinning frequencies of heteronuclear decoupling methods designed to quench rotary resonance. Solid State Nucl. Magn. Reson. 40, 21-26 (2011).

56. Fung, B. M., Khitrin, A. K. \& Ermolaev, K. An improved broadband decoupling sequence for liquid crystals and solids. J. Magn. Reson. 142, 97-101 (2000).

57. Han, B., Liu, Y., Ginzinger, S. W. \& Wishart, D. S. SHIFTX2: significantly improved protein chemical shift prediction. J. Biomol. NMR 50, 43-57 (2011).
58. Drozdetskiy, A., Cole, C., Procter, J. \& Barton, G. J. JPred4: a protein secondary structure prediction server. Nucleic Acids Res. 43, W389-W394 (2015).

\section{Acknowledgements}

We thank Gert Folkers for helpful discussions and Johan van der Zwan for technical support. This work was supported by the Dutch Science Foundation NWO: VENI grant 722.016.002 to S.X., ALW Open program grant 824.15.017 to A.A., NWO-Groot (no. 175.010.2009.002) and TOP-PUNT (no. 718.015.001) grant to M.B. and by uNMR-NL, the National Roadmap Large-Scale NMR Facility of the Netherlands (grant 184.032.207) C.J. is supported by the grants ANR-10-IDEX-0001-02 PSL, ANR-11-LBX-0038, FRM DEQ20170336756. S.B. was supported by the FRM grant FDT201805005465, and CEFIPRA 5703-1, and J.A.S. by the European Union's Horizon 2020 Marie SkłodowskaCurie grant agreement No. 675737, and the FRM grant FDT201904008210.

\section{Author contributions}

Y.L., S.X., A.A., and M.B. designed the experiments, analyzed the data and wrote the paper. Y.L. and P.J.H. produced labeled microtubules with advise from J.A.S. and C.J. Y.L. and S.X. performed and analyzed the NMR experiments; P.J.H. performed the in vitro assays. L.v.B. and F.F. performed the transmission EM experiments. All authors critically reviewed the paper.

\section{Competing interests}

The authors declare no competing interests.

\section{Additional information}

Supplementary information is available for this paper at https://doi.org/10.1038/s41467019-13876-x.

Correspondence and requests for materials should be addressed to A.A. or M.B.

Peer review information Nature Communications thanks the anonymous reviewer(s) for their contribution to the peer review of this work. Peer reviewer reports are available.

Reprints and permission information is available at http://www.nature.com/reprints

Publisher's note Springer Nature remains neutral with regard to jurisdictional claims in published maps and institutional affiliations.

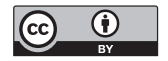

Open Access This article is licensed under a Creative Commons Attribution 4.0 International License, which permits use, sharing, adaptation, distribution and reproduction in any medium or format, as long as you give appropriate credit to the original author(s) and the source, provide a link to the Creative Commons license, and indicate if changes were made. The images or other third party material in this article are included in the article's Creative Commons license, unless indicated otherwise in a credit line to the material. If material is not included in the article's Creative Commons license and your intended use is not permitted by statutory regulation or exceeds the permitted use, you will need to obtain permission directly from the copyright holder. To view a copy of this license, visit http://creativecommons.org/ licenses/by/4.0/.

(C) The Author(s) 2020 